\title{
Maternal Nutritional Imbalance between Linoleic Acid and Alpha-Linolenic Acid Increases Offspring's Anxious Behavior with a Sex-Dependent Manner in Mice
}

\author{
Nobuyuki Sakayori, ${ }^{1,2}$ Hisanori Tokuda, ${ }^{3}$ Kaichi Yoshizaki, ${ }^{1}$ Hiroshi Kawashima, ${ }^{3}$ \\ Sheila M. Innis, ${ }^{4}$ Hiroshi Shibata ${ }^{3}$ and Noriko Osumi ${ }^{1}$ \\ ${ }^{1}$ Department of Developmental Neuroscience, Center for Neuroscience, United Centers for Advanced Research \\ and Translational Medicine, Tohoku University School of Medicine, Sendai, Miyagi, Japan \\ ${ }^{2}$ Research Fellow of the Japan Society for the Promotion of Science, Tokyo, Japan \\ ${ }^{3}$ Institute for Health Care Science, Suntory Wellness Ltd., Soraku-gun, Kyoto, Japan \\ ${ }^{4}$ Department of Pediatrics, Child and Family Research Institute, University of British Columbia, Vancouver, \\ British Columbia, Canada
}

Omega-6 (n-6) and omega-3 (n-3) polyunsaturated fatty acids (PUFAs) are essential nutrients for normal brain development. The principal dietary $n-6$ and $n-3$ PUFAs are linoleic acid (LA) and $\alpha$-linolenic acid (ALA), respectively, We have previously shown that maternal dietary imbalance between these PUFAs, i.e., rich in LA and poor in ALA, affected brain development and increased anxiety-related behavior in the mouse offspring. Here we further addressed sex difference in anxiety-related behavior in the offspring exposed to maternal LA:ALA imbalance. We fed pregnant mice a LA excess/ALA deficient (LA ${ }^{\text {ex }} / A L A^{\text {def }}$ ) diet, and raised their offspring on a well-balanced LA:ALA diet from an early lactation period. When the offspring were grown to adulthood, they were subjected to behavioral and biochemical analyses. We found that both male and female offspring exposed to the LA ${ }^{\text {ex }} / A L A^{\text {def }}$ diet showed increased anxiety-related behavior compared to those exposed to the control diet, which was differently observed between the sexes. The female offspring also exhibited hyperactivity by maternal intake of the LA ${ }^{\mathrm{ex}} / A L A^{\text {def }}$ diet. On the other hand, abnormal depressive behavior was undetected in both sexes. We also found that the ratio of $n-6$ to $n-3$ PUFAs in the brain was unaffected regardless of maternal diet or offspring's sex. Since the $n-6 / n-3$ ratio is known to influence emotional behavior, it is reasonable to assume that LA:ALA imbalance exposed during brain development is the key for causing enhanced anxiety in adulthood. The present study indicates that maternal dietary imbalance between LA and ALA increases offspring's anxiety-related behavior with a sex-dependent manner.

Keywords: anxiety; emotional development; omega-3; omega-6; sex difference Tohoku J. Exp. Med., 2016 September, 240 (1), 31-37. (C) 2016 Tohoku University Medical Press

\section{Introduction}

Polyunsaturated fatty acids (PUFAs) are nutritional components that are essential for normal brain development (Coti Bertrand et al. 2006; Katakura et al. 2009; Sakayori et al. 2011) and function (Carlson et al. 1993; Ryan et al. 2010). PUFAs include omega-6 (n-6) and omega-3 (n-3), and the most principal ones in a diet are linoleic acid (18:2n-6, LA) and $\alpha$-linolenic acid (18:3n-3, ALA), respectively (Elmadfa and Kornsteiner 2009). In many countries, the intake of seed oils, including safflower and soybean oils (which are rich in LA), and that of fish (which are rich in $n-3$ PUFAs) have been increased and decreased, respectively (Blasbalg et al. 2011; Simopoulos et al. 2011). Such a LA excess/ALA deficient $\left(\mathrm{LA}^{\mathrm{ex}} / \mathrm{ALA}^{\mathrm{def}}\right)$ dietary condition is suggested to cause various diseases (Simopoulos 2002).

Emotional state is influenced, at least partially, by a diet. n-3 PUFA-deficiency has been associated with the increased risk of mood and anxiety disorders in humans (Jacka et al. 2013), and increased $n-6$ PUFAs and decreased $n-3$ PUFAs in the brain results in abnormal depressive and anxiety-related behaviors in rodents (Lafourcade et al. 2011; Yamada et al. 2014). Importantly, the intake of $n-3$ PUFAs reduces the risk of depression in females (Colangelo et al. 2009), and females are diagnosed with depression twice as often as males (Kessler et al. 1993); however, little attention is paid to sex difference in the effect of dietary imbalance between LA and ALA on emotional develop-

Received June 28, 2016; revised and accepted August 3, 2016. Published online August 24, 2016; doi: 10.1620/tjem.240.31.

Correspondence: Noriko Osumi, Ph.D., Department of Developmental Neuroscience, Center for Neuroscience, United Centers for Advanced

Research and Translational Medicine, Tohoku University School of Medicine, 2-1 Seiryo-machi, Aoba-ku, Sendai, Miyagi 980-8575, Japan.

e-mail: osumi@med.tohoku.ac.jp 
ment.

We have recently shown that maternal consumption of a $\mathrm{LA}^{\mathrm{ex}} / \mathrm{ALA}^{\mathrm{def}}$ diet caused abnormal brain development and increased anxiety-related behavior in the mouse offspring (Sakayori et al. 2016). However, it is still unknown whether such maternal dietary imbalance causes different behavioral consequences between the male and female offspring and whether such offspring also show abnormal depressive behavior. In the present study, we addressed anxiety-related and depressive behaviors in the male and female offspring exposed to the $\mathrm{LA}^{\mathrm{ex}} / \mathrm{ALA}^{\mathrm{def}}$ condition during brain development. We report that maternal LA:ALA imbalance induced different emotional consequences between the male and female offspring. Overall, this study provides potentially important insights into how a diet affects emotional development.

\section{Materials and Methods}

Animals

Wild-type C57BL/6N mice were obtained from Clea Japan (Tokyo, Japan), and were housed at the Tohoku University School of Medicine. Food and water were available ad libitum. All animal experiments were performed in accordance with the National Institutes of Health Guidelines for the Care and Use of Laboratory Animals, and were approved by the Committee for Animal Experimentation in Tohoku University (\#2013MED-114).

Diets

Virgin female mice at 11 weeks of age were fed one of two semi-purified diets, a control diet or a LA ${ }^{\mathrm{ex}} / \mathrm{ALA}^{\text {def }} \operatorname{diet}$ (Coti Bertrand et al. 2006). Each diet contained a canola oil or a safflower oil, respectively, as a source of lipids. Fatty acid composition of each diet was shown in Table 1. Regarding intake of oleic acid, which was abundant in the control diet, our previous study has shown that maternal intake of the control diet did not increase the level of oleic acid in the embryonic brain compared to that of the $\mathrm{LA}^{\mathrm{ex}} / \mathrm{ALA}^{\mathrm{def}}$ diet (Sakayori et al. 2016), suggesting that oleic acid in these diets did not

Table 1. Fatty acid composition.

\begin{tabular}{lrr}
\hline fatty acid & \multicolumn{1}{c}{ control } & LAex/ALAdef \\
\hline $16: 0$ & $5.2 \%$ & $7.6 \%$ \\
$18: 0$ & $1.9 \%$ & $1.6 \%$ \\
total SFAs & $8.2 \%$ & $9.9 \%$ \\
\hline $18: 1 n-9$ & $59.6 \%$ & $15.1 \%$ \\
$20: 1 n-9$ & $1.4 \%$ & $0.2 \%$ \\
total MUFAs & $61.5 \%$ & $15.4 \%$ \\
\hline $18: 2 n-6$ & $20.7 \%$ & $74.3 \%$ \\
total $n$-6 PUFAs & $20.8 \%$ & $74.4 \%$ \\
\hline $18: 3 n-3$ & $9.4 \%$ & $0.3 \%$ \\
total $n$-3 PUFAs & $9.4 \%$ & $0.3 \%$ \\
\hline
\end{tabular}

Fatty acid composition in the diets. Fatty acids with more than $1 \%$ in either diet are shown. Levels of fatty acids are expressed as the percentage of total lipids. The systematic name of each fatty acid is palmitic acid (16:0), stearic acid (18:0), oleic acid $(18: 1 n-9)$, eicosenoic acid (20:1n-9), linoleic acid (18:2n-6), or $\alpha$-linolenic acid (18:3n-3). have important roles in brain development. After two weeks, mice were mated, and pregnant mice were maintained on their respective diet through gestation and the first 10 days of lactation. Thereafter, mother mice were fed the AIN-93G diet (Reeves et al. 1993) (Oriental Yeast, Tokyo, Japan) during lactation, and after weaning, the offspring were directly fed the AIN-93M diet (Reeves et al. 1993) (Oriental Yeast).

\section{Behavioral tests}

The offspring at 13-15 weeks of age were subjected to behavioral tests with following order. Each test was performed between 10:00 a.m. and 6:00 p.m. (one test per day).

The open field test, which evaluates locomotor activity and anxiety-related behavior, was conducted with the apparatus consisting of a transparent floor $(50 \mathrm{~cm} \times 50 \mathrm{~cm})$ with 30 -cm-high black walls. The floor was divided into the center zone (the central $30 \mathrm{~cm} \times 30$ $\mathrm{cm}$ ) and the peripheral zone (the other area) under 1,160 lx brightness. Each mouse was placed in the center of the open field and recorded its free exploration trajectory for 10 min using a video tracking system (ANY-maze; Stoelting, Wood Dale, IL). The time spent in the center zone, the number of entries into the center zone, and the total distance traveled were measured.

The elevated plus maze test, which evaluates locomotor activity and anxiety-related behavior, was conducted with the apparatus consisting of a plus-shaped maze elevated $47 \mathrm{~cm}$ above the floor, containing two opposing open arms $(30 \mathrm{~cm} \times 5 \mathrm{~cm}$ each $)$ with a $1-\mathrm{cm}$-high clear Plexiglas edge surrounded the arms to prevent falls and two opposing arms enclosed by 30 -cm-high walls with an open top. Each mouse was placed in the center of the maze facing the open arm and recorded its free exploration trajectory for 5 min using a video tracking system (ANY-maze; Stoelting). The times spent in the open and closed arms and the total distance traveled were measured.

For the Porsolt forced swimming test, which evaluates depressive behavior (Porsolt et al. 2001), a cylinder (15-cm-diameter and 25 -cm-high) filled with water $\left(25 \pm 1{ }^{\circ} \mathrm{C}\right)$ to a height of $15 \mathrm{~cm}$ was used. Each mouse was exposed to swim stress for $2 \mathrm{~min}$, which was based on the previous study (Lafourcade et al. 2011), and was monitored from the side by a video camera. The time spent immobile was measured. A mouse was considered to be immobile when it made only minimal movements to keep head above water.

\section{Fatty acid analysis of brain lipid fractions}

Fatty acid analysis was performed according to the method (Sakayori et al. 2016). One day after behavioral analyses, the offspring maintained under free-feeding condition were deeply anesthetized. The brains were dissected from the surrounding tissue, total lipids were extracted from the flash-frozen brains using chloroform and methanol extraction, and fatty acid residues in the total lipids were subsequently analyzed. An internal standard (pentadecanoic acid) was added to each sample, and each sample was incubated in methanolic $\mathrm{HCl}$ at $50^{\circ} \mathrm{C}$ for 3 hours to induce the transmethylation of fatty acid residues. Capillary gas-liquid chromatography with $n$-hexane was performed to extract the fatty acid methyl esters. The analytical conditions were as follows: the gas chromatograph was an Agilent 6890 (Agilent Technologies, Santa Clara, CA); the column was an SP-2330 $(30 \mathrm{~m} \times 0.32 \mathrm{~mm} \times 0.2 \mu \mathrm{m}$; Supelco, Bellefonte, $\mathrm{PA}$ ); the carrier was $\mathrm{He}$ (at $30 \mathrm{~cm} / \mathrm{sec}$ ); and the initial column temperature was $180^{\circ} \mathrm{C}$ for $2 \mathrm{~min}$, after which column temperature increased to $220^{\circ} \mathrm{C}$ at a rate of $2^{\circ} \mathrm{C} / \mathrm{min}$. 


\section{Statistical analysis}

Two-way factorial analysis of variance (ANOVA) (except for Fig. 2B) or three-way ANOVA (time as repeated measure) (for Fig. 2B) was performed. Post hoc Bonferroni method was used to compare each group when a statistically significant difference was observed in the interaction between the dietary effect and the sexual effect in two-way factorial ANOVA. All data are expressed as the mean \pm SEM. In the behavioral analyses, 11 or 12 male offspring in the control group or the $\mathrm{LA}^{\mathrm{ex}} / \mathrm{ALA}^{\mathrm{def}}$ group, respectively, and 11 female offspring in both control and $\mathrm{LA}^{\mathrm{ex}} / \mathrm{ALA}^{\mathrm{def}}$ groups were used.

\section{Results}

Maternal dietary imbalance of PUFAs does not affect offspring's food intake or body weight

Before examination of offspring's behavior, we analyzed food intake and body weight of the offspring, and found that maternal diet affected neither of the factors (Fig. 1A, B), although body weight of the male offspring was heavier than that of the female (Fig. 1B). We have also confirmed that both food intake and body weight of the mother mice were unaffected by the same diet as shown in our previous study (Sakayori et al. 2016). These data indicate that maternal LA:ALA imbalance does not cause notable hypoplasia or obesity in their offspring.
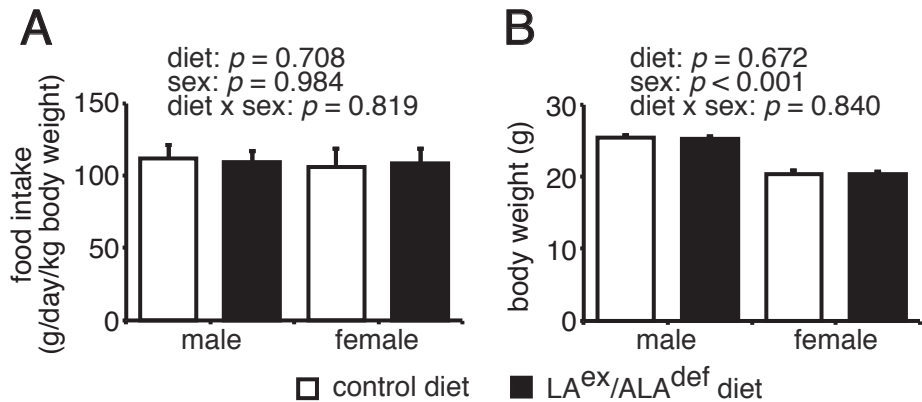

Fig. 1. Evaluation of food intake and body weight

Food intake (A) and body weight (B) of the adult offspring before behavioral analyses were measured. (A) $n=5$ per each group. (B) $n=11$ or 12 male offspring in the control group or the LA ${ }^{\text {ex }} / \mathrm{ALA}^{\text {def }}$ group, respectively, and $n=11$ female offspring in both control and $\mathrm{LA}^{\mathrm{ex}} / \mathrm{ALA}^{\mathrm{def}}$ groups.
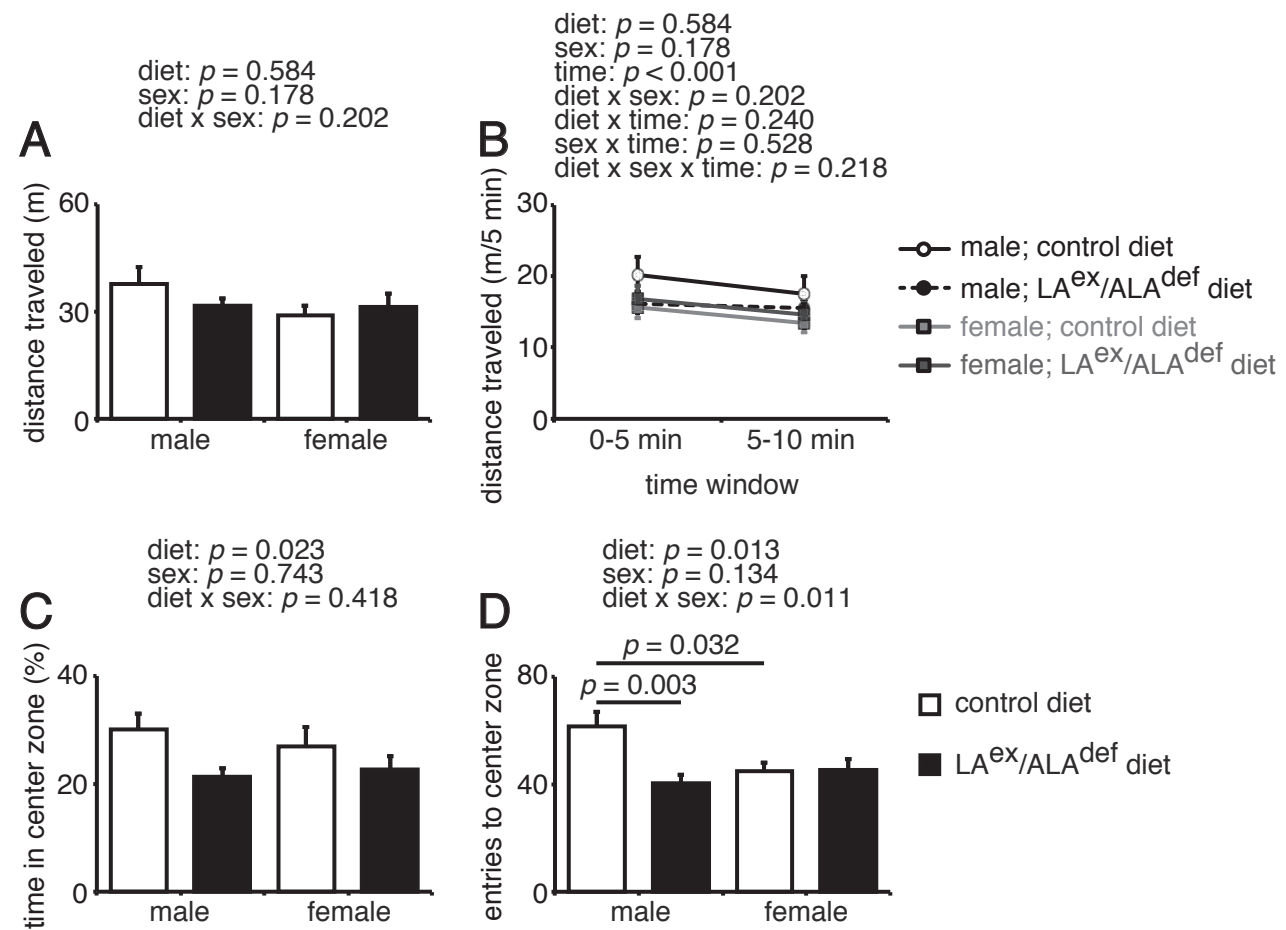

Fig. 2. Evaluation of basal locomotor activity and anxiety-related behavior in the open field test.

Distance traveled (A and B), time spent in the center zone (C), and the number of entries into the center zone (D) were measured. 

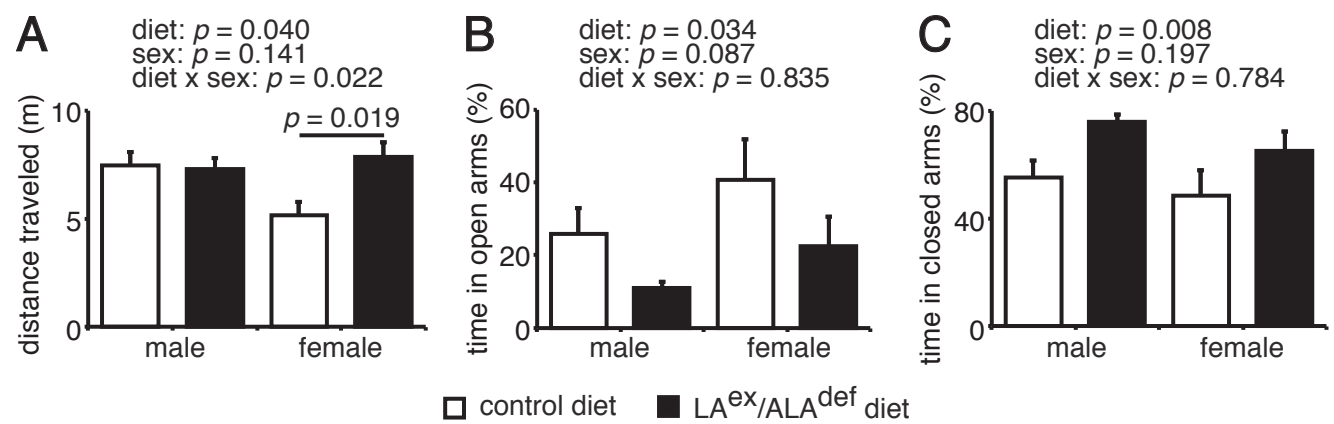

Fig. 3. Evaluation of basal locomotor activity and anxiety-related behavior in the elevated plus maze test. Distance traveled (A) and time spent in the open (B) or closed (C) arms were measured.

Maternal dietary imbalance of PUFAs increases offspring's anxiety-related behavior with a sex-dependent manner

We next examined locomotor activity of these offspring in the open field test (Fig. 2) and in the elevated plus maze test (Fig. 3). In the open field test, the offspring showed similar distance traveled regardless of maternal diet or their sex (Fig. 2A). Distance traveled during first or last 5 min was also unaffected (Fig. 2B). These data suggest that maternal consumption of the LA ${ }^{\text {ex }} / \mathrm{ALA}^{\text {def }}$ diet did not affect locomotor activity in their offspring in this analysis. However, the female offspring born to dams fed the LA ${ }^{\mathrm{ex}}$ / ALA $^{\text {def }}$ diet had increased distance traveled than those born to dams fed the control diet in the elevated plus maze test (Fig. 3A). These data imply that maternal consumption of the $\mathrm{LA}^{\mathrm{ex}} / \mathrm{ALA}^{\mathrm{def}}$ diet may induce hyperactivity in the female offspring depending on the behavioral test.

We next evaluated anxiety-related behavior using the open field test. Regarding time spent in the center zone, the offspring born to dams fed the LA ${ }^{\mathrm{ex}} / \mathrm{ALA}^{\text {def }}$ diet spent less time in the center zone compared to those born to dams fed the control diet, regardless of their sex (Fig. 2C). On the contrary, the female offspring born to dams fed the control diet had fewer entries into the center zone than the male offspring born to dams fed the control diet, and the male offspring born to dams fed the LA ${ }^{\mathrm{ex}} / \mathrm{ALA}^{\mathrm{def}}$ diet also had fewer entries into the center zone than the male offspring born to dams fed the control diet (Fig. 2D). Thus, there is a sex difference in the vulnerability for the effect of maternal LA:ALA imbalance on offspring's anxiety-related behavior when evaluated by the open field test; both male and female offspring exposed to the $\mathrm{LA}^{\mathrm{ex}} / \mathrm{ALA}^{\text {def }}$ diet show less time spent in the center zone compared to those exposed to the control diet, but the male offspring exposed to the $\mathrm{LA}^{\mathrm{ex}} /$ ALA $^{\text {def }}$ diet also show fewer entries into the center zone than those exposed to the control diet.

Based on the above findings, we further evaluated anxiety-related behavior using a different behavioral paradigm, i.e., the elevated plus maze test. In this case, both male and female offspring born to dams fed the $\mathrm{LA}^{\mathrm{ex}} /$ $\mathrm{ALA}^{\text {def }}$ diet spent less time in the open arms and more time in the closed arms compared to those born to dams fed the

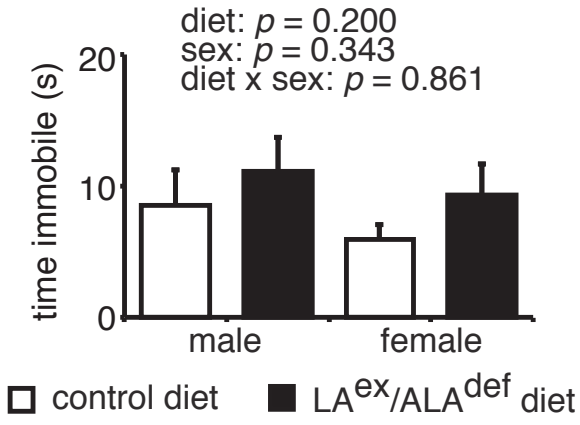

Fig. 4. Evaluation of depressive behavior in the Porsolt forced swimming test. Time spent immobile was measured.

control diet (Fig. 3B, C). These data indicate that increased anxiety-related behavior in the offspring caused by maternal consumption of the $\mathrm{LA}^{\mathrm{ex}} / \mathrm{ALA}^{\mathrm{def}}$ diet is observed regardless of their sex when evaluated by the elevated plus maze test.

As another text for emotional behavior, the Porsolt forced swimming test was performed to evaluate depression. There was no difference in the time spent immobile regardless of maternal diet or their sex (Fig. 4). Thus, mice exposed to the $\mathrm{LA}^{\mathrm{ex}} / \mathrm{ALA}^{\mathrm{def}}$ condition during brain development do not exhibit abnormal depressive behavior.

Maternal dietary imbalance of PUFAs does not cause n-6:n-3 imbalance in the adult offspring's brain

How did maternal consumption of the LA ${ }^{\mathrm{ex}} / \mathrm{ALA}^{\text {def }}$ diet affect emotion of their offspring? Dietary LA and ALA are metabolized and detected as arachidonic acid (ARA, 20:4n-6) and docosahexaenoic acid (DHA, 22:6n-3), respectively, in the brain (Sastry 1985). We have previously shown that maternal intake of the $\mathrm{LA}^{\mathrm{ex}} / \mathrm{ALA}^{\text {def }}$ diet increased the level of ARA and decreased that of DHA in the embryonic and postnatal offspring's brains (Sakayori et al. 2016). Importantly, the ratio of $n-6$ to $n$-3 PUFAs in the adult brain shows positive correlation with fear memory (Yamada et al. 2014). Based on these reports, it is possible that $n-6: n-3$ imbalance in the developing brain persisted into the adult brain, which consequently affected anxiety.

Here, we examined the level of fatty acids in the adult brain of the offspring using capillary gas-liquid chromatog- 
Table 2. Fatty acids in the adult brain of the offspring.

\begin{tabular}{|c|c|c|c|c|c|c|c|}
\hline \multirow{2}{*}{ fatty acid } & \multicolumn{2}{|c|}{ male } & \multicolumn{2}{|c|}{ female } & \multicolumn{3}{|c|}{ two-way factorial ANOVA } \\
\hline & control diet & LAex/ALAdef diet & control diet & LAex/ALAdef diet & dietary effect & sexual effect & interaction \\
\hline 16:0 & $18.99 \pm 0.07$ & $18.89 \pm 0.16$ & $18.92 \pm 0.16$ & $19.06 \pm 0.17$ & $F(1,8)=0.010, p=0.923$ & $F(1,8)=0.121, p=0.737$ & $F(1,8)=0.694, p=0.429$ \\
\hline 18:0 & $20.53 \pm 0.05$ & $20.53 \pm 0.06$ & $20.65 \pm 0.04$ & $20.79 \pm 0.04$ & $F(1,8)=1.980, p=0.197$ & $F(1,8)=13.675, p=0.006$ & $F(1,8)=1.896, p=0.206$ \\
\hline total SFAs & $40.45 \pm 0.07$ & $40.42 \pm 0.18$ & $40.53 \pm 0.16$ & $40.77 \pm 0.15$ & $F(1,8)=0.555, p=0.478$ & $F(1,8)=2.301, p=0.168$ & $F(1,8)=0.855, p=0.382$ \\
\hline $18: 1 n-7$ & $4.96 \pm 0.11$ & $4.89 \pm 0.05$ & $4.78 \pm 0.08$ & $4.68 \pm 0.07$ & $F(1,8)=1.093, p=0.326$ & $F(1,8)=5.785, p=0.043$ & $F(1,8)=0.027, p=0.875$ \\
\hline $18: 1 n-9$ & $16.54 \pm 0.05$ & $16.45 \pm 0.06$ & $16.64 \pm 0.13 *$ & $16.01 \pm 0.17^{*}$ & $F(1,8)=9.667, p=0.014$ & $F(1,8)=2.164, p=0.179$ & $F(1,8)=5.624, p=0.045$ \\
\hline $20: 1 n-9$ & $1.99 \pm 0.21$ & $2.12 \pm 0.23$ & $2.06 \pm 0.05$ & $1.89 \pm 0.21$ & $F(1,8)=0.596, p=0.462$ & $F(1,8)=0.043, p=0.841$ & $F(1,8)=1.873, p=0.208$ \\
\hline $24: 1 n-9$ & $1.12 \pm 0.08$ & $1.11 \pm 0.07$ & $1.07 \pm 0.06$ & $0.98 \pm 0.05$ & $F(1,8)=0.530, p=0.488$ & $F(1,8)=1.538, p=0.250$ & $F(1,8)=0.347, p=0.572$ \\
\hline total MUFAs & $25.14 \pm 0.21$ & $25.04 \pm 0.16$ & $25.11 \pm 0.39$ & $24.01 \pm 0.41$ & $F(1,8)=3.684, p=0.091$ & $F(1,8)=2.815, p=0.132$ & $F(1,8)=2.537, p=0.150$ \\
\hline $20: 4 n-6$ & $10.66 \pm 0.18$ & $10.55 \pm 0.03$ & $10.60 \pm 0.10$ & $10.78 \pm 0.09$ & $F(1,8)=0.109, p=0.750$ & $F(1,8)=0.545, p=0.481$ & $F(1,8)=1.556, p=0.248$ \\
\hline $22: 4 n-6$ & $2.80 \pm 0.07$ & $2.91 \pm 0.06$ & $2.81 \pm 0.07$ & $2.90 \pm 0.03$ & $F(1,8)=2.400, p=0.160$ & $F(1,8)=0.001, p=0.979$ & $F(1,8)=0.044, p=0.839$ \\
\hline total $n$-6 PUFAs & $15.24 \pm 0.17$ & $15.37 \pm 0.06$ & $14.97 \pm 0.10$ & $15.39 \pm 0.03$ & $F(1,8)=7.278, p=0.027$ & $F(1,8)=1.520, p=0.253$ & $F(1,8)=2.204, p=0.176$ \\
\hline $22: 6 n-3$ & $18.89 \pm 0.12$ & $18.90 \pm 0.04$ & $19.11 \pm 0.26$ & $19.56 \pm 0.27$ & $F(1,8)=1.395, p=0.272$ & $F(1,8)=4.957, p=0.057$ & $F(1,8)=1.196, p=0.306$ \\
\hline total $n$-3 PUFAs & $19.05 \pm 0.13$ & $19.06 \pm 0.03$ & $19.28 \pm 0.26$ & $19.72 \pm 0.27$ & $F(1,8)=1.295, p=0.288$ & $F(1,8)=4.927, p=0.057$ & $F(1,8)=1.162, p=0.312$ \\
\hline ratio of $n-6$ to $n-3$ & $0.80 \pm 0.01$ & $0.78 \pm 0.01$ & $0.81 \pm 0.00$ & $0.78 \pm 0.01$ & $F(1,8)=0.236, p=0.640$ & $F(1,8)=5.272, p=0.051$ & $F(1,8)=0.005, p=0.945$ \\
\hline
\end{tabular}

Fatty acid composition in the adult brain. Fatty acids with more than $1 \%$ in the brain are shown. $n=3$ brains per each group. ${ }^{*} p<0.05$ by the post hoc Bonferroni method. Levels of fatty acids are expressed as the percentage of total lipids. Ratio of $n-6$ to $n-3$ means the ratio of total $n$-6 PUFAs to total $n$-3 PUFAs. The systematic name of each fatty acid is palmitic acid (16:0), stearic acid (18:0), vaccenic acid (18:1n-7), oleic acid (18:1n-9), eicosenoic acid (20:1n-9), nervonic acid (24:1n-9), arachidonic acid (20:4n-6), adrenic acid (22:4n6), or docosahexaenoic acid (22:6n-3).

raphy. Interestingly, no significant difference, especially in the levels of ARA and DHA and in the ratio of $n-6$ to $n-3$ PUFAs, was detectable in the adult brain regardless of maternal diet or their sex (Table 2). Two-way factorial ANOVA indicated only slight differences (less than 1\%) in the levels of stearic acid (18:0) and vaccenic acid (18:1n-7) between males and females, in the level of total $n-6$ PUFAs between the offspring born to dams fed the control diet or the LA $^{\text {ex }} /$ ALA $^{\text {def }}$ diet, and in the level of oleic acid (18:1n-9) between the female offspring born to dams fed the control diet and the female offspring born to dams fed the $\mathrm{LA}^{\mathrm{ex}} /$ ALA $^{\text {def }}$ diet. Thus, it may be possible that increased anxiety-related behavior with sex difference is due to developmental effects of $n-6: n-3$ imbalance rather than the fatty acid profile in the adult brain. These results support the notion that LA:ALA imbalance during brain development rather than that in the adult brain is a cause for enhanced anxiety.

\section{Discussion}

We, in the present study, analyzed the sex difference in the effect of maternal LA:ALA imbalance on the anxietyrelated behavior of the offspring. Interaction between maternal diet and their sex was detected in the number of entries to the center zone in the open field test (Fig. 2D). Especially, the female offspring exposed to the control diet show fewer entries into the center zone than the male offspring exposed to the control diet. Due to this behavioral difference between the sexes, the impact of maternal LA:ALA imbalance on the number of entry into the center zone might be ineffective in the female offspring. Thus, these data show that maternal LA:ALA imbalance affects emotional development differently between offspring's sex depending on the behavioral context. A previous report suggested a higher risk of depression in females (Kessler et al. 1993). Our findings, however, consider it unlikely to be caused by maternal LA:ALA imbalance. The present study still emphasizes the importance to analyze the impact of a diet on emotional development by taking sex difference into account.

Sex difference of anxiety-related behavior in the open field test might be confounded by stress-vulnerability. The fact that the $\mathrm{C} 57 \mathrm{BL} / 6 \mathrm{~N}$ female mice show fewer entries into the center zone than the C57BL/6N male mice in our study (Fig. 2D) seems to be inconsistent with a previous report, which has shown that $\mathrm{C} 57 \mathrm{BL} / 6 \mathrm{~J}$ female mice have a similar anxiety level in the open field test as $\mathrm{C} 57 \mathrm{BL} / 6 \mathrm{~J}$ male mice (An et al. 2011). One of the obvious differences between our study and their study is brightness of the floor in the open field test; in our study it was at intensity of $1,160 \mathrm{~lx}$, but $400 \mathrm{~lx}$ in their study. It is thus possible that female mice might be more vulnerable for light stress than male mice, consequently showing increased anxiety-related behavior compared to male mice in our study. To investigate this hypothesis, it is necessary to evaluate sex difference in vulnerability for light stress using C57BL/6N mice in the open field test with several brightness of the floor.

Anxiety induced by maternal LA:ALA imbalance might also be affected differently by the age, which should be taken into consideration in the behavioral analyses. Several researchers (Nakashima et al. 1993; Frances et al. 1995; Carrie et al. 2000) fed mother mice a LA ${ }^{\mathrm{ex}} / \mathrm{ALA}^{\mathrm{def}}$ diet before mating through gestation and lactation. After weaning, they fed the offspring the same diet as their dams fed, and performed the elevated plus maze test. In this paradigm, increased anxiety-related behavior was not detected at 6-8 weeks of age (Nakashima et al. 1993; Frances et al. 1995), but observed at 4-5 months of age (Carrie et al. 2000). These reports suggest that enhanced anxiety following maternal LA:ALA imbalance become obvious in adulthood but not fully appeared during the young stages. We thus performed the behavioral analyses using the offspring at 13-15 weeks of age.

When is the abnormal depressive behavior established 
after maternal exposure to LA:ALA imbalance? A previous report has shown that maternal consumption of a peanut oil-containing diet (rich in LA but poor in ALA) from fertilization through postnatal day 21 (P21) increased the time spent immobile in the Porsolt forced swimming test measured for 2 minutes (Lafourcade et al. 2011). In the present study, we exposed the $\mathrm{LA}^{\mathrm{ex}} / \mathrm{ALA}^{\text {def }}$ diet from fertilization through P10, and performed the same forced swimming test in the same condition (Fig. 4). Taken these studies together, it may be possible that the onset of depression following maternal consumption of the $\mathrm{LA}^{\mathrm{ex}} / \mathrm{ALA}^{\text {def }}$ diet is determined during the lactation period, especially from P11 through P21. Importance of maternal nutritional state in the lactation period is also noticed in a recent report using mice; feeding dams a high fat diet during the lactation period inhibits the neuronal projection of several hypothalamic areas in offspring (Vogt et al. 2014). Detailed studies are further required to understand critical developmental events during the lactation period that cause depression.

High comorbidity rates of depressive disorders are noticed in patients with anxiety disorders (Lamers et al. 2011). Our study shows increased anxiety-related behavior without abnormal depressive behavior in mice. This could give important insights on the critical period for mental illness; maternal LA:ALA imbalance during the prenatal and early postnatal stages may cause anxiety-related behavior in the offspring, while depression may be induced by maternal PUFA imbalance at later stages. In other words, increased anxiety caused by maternal LA:ALA imbalance may be developmentally independent with the onset of depression. However, if maternal PUFA imbalance continues, the offspring may suffer from both anxiety and depression. It is of note that massive neurogenesis continues to the early postnatal stage in certain brain regions including the hippocampus (Sugiyama et al. 2013). Decreased neurogenesis in the hippocampus is considered to relate with mood disorders (Eisch and Petrik 2012). Further studies are necessary to reveal responsible brain regions and molecular/cellular mechanisms of the onset of mental illness caused by maternal malnutrition.

Maternal LA:ALA imbalance also induced hyperlocomotion in female offspring mice (Fig. 3A). This result is interesting in regard with a common neurodevelopmental disorder, i.e., attention deficit hyperactivity disorder (ADHD). Consistent with our data, hyperactivity in mice and rats induced by consumption of $n-3$ PUFA-deficient diets are repeatedly reported (reviewed by Fedorova and Salem 2006). Spontaneously hypertensive rats are considered to be an animal model for ADHD (Sagvolden and Sergeant 1998), and exhibit a decreased level of DHA in the brain (Wei et al. 1987). So far, the relationship between LA:ALA imbalance during brain development and the onset of ADHD is not well focused. It will be interesting to more precisely evaluate effects of PUFAs on various behavioral paradigms for ADHD as well as for anxiety disorders.

\section{Acknowledgments}

We express our deepest condolences to late S.M. Innis. We thank Dr. R.A. Dyer for preparation of the diets; Dr. Y. Iguchi for advice in statistical analyses; and Ms. K. Ueda, J. Yoon, E. Kiryu, E. Otsuki, S. Makino, and A. Ogasawara and Mr. K. Koike for animal care. This work was supported by a Grant-inAid for JSPS Fellows (N.S.), by a Grant-in-Aid for Scientific Research (B) from MEXT (\#21300115, N.O.), and by a Research Grant from the Asahi Glass Foundation (N.O.).

\section{Conflict of Interest}

H.T., H.K., and H.S. are employees of Suntory Wellness Ltd., a company that markets health-food products; their employment did not affect their compliance with all relevant scientific and ethical guidelines. The remaining authors also declare no conflict of interest.

\section{References}

An, X.L., Zou, J.X., Wu, R.Y., Yang, Y., Tai, F.D., Zeng, S.Y., Jia, R., Zhang, X., Liu, E.Q. \& Broders, H. (2011) Strain and sex differences in anxiety-like and social behaviors in C57BL/6J and BALB/cJ mice. Exp. Anim., 60, 111-123.

Blasbalg, T.L., Hibbeln, J.R., Ramsden, C.E., Majchrzak, S.F. \& Rawlings, R.R. (2011) Changes in consumption of omega-3 and omega- 6 fatty acids in the United States during the 20th century. Am. J. Clin. Nutr., 93, 950-962.

Carlson, S.E., Werkman, S.H., Peeples, J.M., Cooke, R.J. \& Tolley, E.A. (1993) Arachidonic acid status correlates with first year growth in preterm infants. Proc. Natl. Acad. Sci. USA, 90, 1073-1077.

Carrie, I., Clement, M., de Javel, D., Frances, H. \& Bourre, J.M. (2000) Phospholipid supplementation reverses behavioral and biochemical alterations induced by $n-3$ polyunsaturated fatty acid deficiency in mice. J. Lipid Res., 41, 473-480.

Colangelo, L.A., He, K., Whooley, M.A., Daviglus, M.L. \& Liu, K. (2009) Higher dietary intake of long-chain omega-3 polyunsaturated fatty acids is inversely associated with depressive symptoms in women. Nutrition, 25, 1011-1019.

Coti Bertrand, P., O'Kusky, J.R. \& Innis, S.M. (2006) Maternal dietary (n-3) fatty acid deficiency alters neurogenesis in the embryonic rat brain. J. Nutr., 136, 1570-1575.

Eisch, A.J. \& Petrik, D. (2012) Depression and hippocampal neurogenesis: a road to remission? Science, 338, 72-75.

Elmadfa, I. \& Kornsteiner, M. (2009) Dietary fat intake: a global perspective. Ann. Nutr. Metab., 54 Suppl 1, 8-14.

Fedorova, I. \& Salem, N. Jr. (2006) Omega-3 fatty acids and rodent behavior. Prostaglandins Leukot. Essent. Fatty Acids, 75, 271-289.

Frances, H., Monier, C. \& Bourre, J.M. (1995) Effects of dietary alpha-linolenic acid deficiency on neuromuscular and cognitive functions in mice. Life Sci., 57, 1935-1947.

Jacka, F.N., Pasco, J.A., Williams, L.J., Meyer, B.J., Digger, R. \& Berk, M. (2013) Dietary intake of fish and PUFA, and clinical depressive and anxiety disorders in women. Br. J. Nutr., 109, 2059-2066.

Katakura, M., Hashimoto, M., Shahdat, H.M., Gamoh, S., Okui, T., Matsuzaki, K. \& Shido, O. (2009) Docosahexaenoic acid promotes neuronal differentiation by regulating basic helixloop-helix transcription factors and cell cycle in neural stem cells. Neuroscience, 160, 651-660.

Kessler, R.C., McGonagle, K.A., Swartz, M., Blazer, D.G. \& Nelson, C.B. (1993) Sex and depression in the National Comorbidity Survey. I: lifetime prevalence, chronicity and recurrence. J. Affect. Disord., 29, 85-96.

Lafourcade, M., Larrieu, T., Mato, S., Duffaud, A., Sepers, M., Matias, I., De Smedt-Peyrusse, V., Labrousse, V.F., Bretillon, 
L., Matute, C., Rodriguez-Puertas, R., Laye, S. \& Manzoni, O.J. (2011) Nutritional omega-3 deficiency abolishes endocannabinoid-mediated neuronal functions. Nat. Neurosci., 14, 345-350.

Lamers, F., van Oppen, P., Comijs, H.C., Smit, J.H., Spinhoven, P., van Balkom, A.J., Nolen, W.A., Zitman, F.G., Beekman, A.T. \& Penninx, B.W. (2011) Comorbidity patterns of anxiety and depressive disorders in a large cohort study: the Netherlands Study of Depression and Anxiety (NESDA). J. Clin. Psychiatry, 72, 341-348.

Nakashima, Y., Yuasa, S., Hukamizu, Y., Okuyama, H., Ohhara, T., Kameyama, T. \& Nabeshima, T. (1993) Effect of a high linoleate and a high alpha-linolenate diet on general behavior and drug sensitivity in mice. J. Lipid Res., 34, 239-247.

Porsolt, R.D., Brossard, G., Hautbois, C. \& Roux, S. (2001) Rodent models of depression: forced swimming and tail suspension behavioral despair tests in rats and mice. Curr. Protoc. Neurosci., Unit 8.10A.

Reeves, P.G., Nielsen, F.H. \& Fahey, G.C. Jr. (1993) AIN-93 purified diets for laboratory rodents: final report of the American Institute of Nutrition ad hoc writing committee on the reformulation of the AIN-76A rodent diet. J. Nutr., 123, 19391951.

Ryan, A.S., Astwood, J.D., Gautier, S., Kuratko, C.N., Nelson, E.B. \& Salem, N. Jr. (2010) Effects of long-chain polyunsaturated fatty acid supplementation on neurodevelopment in childhood: a review of human studies. Prostaglandins Leukot. Essent. Fatty Acids, 82, 305-314.

Sagvolden, T. \& Sergeant, J.A. (1998) Attention deficit/hyperactivity disorder: from brain dysfunctions to behaviour. Behav. Brain Res., 94, 1-10.

Sakayori, N., Kikkawa, T., Tokuda, H., Kiryu, E., Yoshizaki, K., Kawashima, H., Yamada, T., Arai, H., Kang, J.X., Katagiri, H., Shibata, H., Innis, S.M., Arita, M. \& Osumi, N. (2016)
Maternal dietary imbalance between omega- 6 and omega- 3 polyunsaturated fatty acids impairs neocortical development via epoxy metabolites. Stem Cells, 34, 470-482.

Sakayori, N., Maekawa, M., Numayama-Tsuruta, K., Katura, T., Moriya, T. \& Osumi, N. (2011) Distinctive effects of arachidonic acid and docosahexaenoic acid on neural stem/progenitor cells. Genes Cells, 16, 778-790.

Sastry, P.S. (1985) Lipids of nervous tissue: composition and metabolism. Prog. Lipid Res., 24, 69-176.

Simopoulos, A.P. (2002) The importance of the ratio of omega-6/ omega-3 essential fatty acids. Biomed. Pharmacother, 56, 365-379.

Simopoulos, A.P., Faergeman, O. \& Bourne, P.G. (2011) Action plan for a healthy agriculture, healthy nutrition, healthy people. J. Nutrigenet. Nutrigenomics, 4, 65-68.

Sugiyama, T., Osumi, N. \& Katsuyama, Y. (2013) The germinal matrices in the developing dentate gyrus are composed of neuronal progenitors at distinct differentiation stages. Dev. Dyn., 242, 1442-1453.

Vogt, M.C., Paeger, L., Hess, S., Steculorum, S.M., Awazawa, M., Hampel, B., Neupert, S., Nicholls, H.T., Mauer, J., Hausen, A.C., Predel, R., Kloppenburg, P., Horvath, T.L. \& Bruning, J.C. (2014) Neonatal insulin action impairs hypothalamic neurocircuit formation in response to maternal high-fat feeding. Cell, 156, 495-509.

Wei, J.W., Yang, L.M., Sun, S.H. \& Chiang, C.L. (1987) Phospholipids and fatty acid profile of brain synaptosomal membrane from normotensive and hypertensive rats. Int. J. Biochem., 19, 1225-1228.

Yamada, D., Takeo, J., Koppensteiner, P., Wada, K. \& Sekiguchi, M. (2014) Modulation of Fear Memory by Dietary Polyunsaturated Fatty Acids via Cannabinoid Receptors. Neuropsychopharmacology, 39, 1852-1860. 